\title{
Ultrastructural AFM Markers of Endogenous Intoxication in Community-Acquired Pneumonia
}

\author{
DOI: $10.17691 / \mathrm{stm} 2017.9 .2 .06$ \\ Received April 14, 2016
}

V.N. Kotelnikov, MD, DSc, Chief'; Professor, Military Training Centre ${ }^{2}$;

A.A. Karpenko, PhD, Senior Researcher, Cell Biophysics Laboratory3;

A.P. Kim, MD, PhD, Chief4;

B.I. Geltser, MD, DSc, Professor, Corresponding Member of the Russian Academy of Sciences, Director of the Department of Fundamental and Clinical Medicine, Biomedicine School ${ }^{5}$

${ }^{1}$ Far Eastern Branch of State Research Experimental Institute of Military Medicine, 100d Borisenko St., Vladivostok, 690080, Russian Federation;

2Pacific State Medical University, 2 Ostryakov Avenue, Vladivostok, 690002, Russian Federation;

${ }^{3}$ National Research Center of Marine Biology of Far-Eastern Division of the Russian Academy of Science, 17 Pal'chevsky St., Vladivostok, 690041, Russian Federation;

${ }^{4} 439$ Military Hospital, Ministry of Defence of the Russian Federation, 7 Karbyshev St., Ussuriysk, 692511, Russian Federation;

${ }^{5}$ Far Eastern Federal University, 8 Sukhanov St., Vladivostok, 690091, Russian Federation

The aim of the investigation was to assess the information content of the parameters of atomic force microscopy to characterize the severity of endogenous intoxication in community-acquired pneumonia according to the findings of ultrastructural parameters of red blood cells.

Materials and Methods. Using atomic force microscopy we studied nanoanatomical and micromechanical indices of red blood cells in 71 patients with severe and mild community-acquired pneumonia aged 18-23 (mean age: 20.4 \pm 0.6 years) against the background of endogenous intoxication of varying severity. The control group consisted of 20 volunteers. Red blood cell samples were taken on admission, and on days 3, 7 and 14 after hospitalization. To determine their morphological features, a contact mode of nanomechanical mapping was used. Endogenous intoxication severity was assessed according to standard clinical and laboratory parameters including hematological, biochemical and immunological markers.

Results. We obtained the reference values of micromechanical parameters of erythrocyte membranes in healthy young subjects and in patients with community-acquired pneumonia of varying severity of endogenous intoxication. The values can serve as a guide for future researches in similar samplings. Viscoelasticity properties of erythrocyte membranes in the disease were found to be closely related to endotoxemia intensity. In disease progression, the elasticity modulus and adhesion force of erythrocyte membrane decreased by 5.2 and 2.4, respectively, while the strain increased by 2.6. A correlation analysis revealed the presence of strong interrelations between the parameters characterizing endogenous intoxication severity (average-weight molecules, IL-10, tumor necrosis factor, leukocyte, nuclear, hematological indices of intoxication, etc.), and micromechanical parameters of cell membranes. The indices of geometric "portrait" of red blood cells (area, diameter, rim height, etc.) did not differ significantly indicating the stability of their cytoskeleton.

Conclusion. The studies prove the information content of micromechanical parameters of erythrocyte membranes by atomic force microscopy to characterize the severity of endogenous intoxication in community-acquired pneumonia. The findings complement the presentation of pathophysiological sequelae of endogenous intoxication, their effect on the ultrastructure of cells and adaptive nature of the changes in micromechanical properties of membranes.

Key words: endogenous intoxication markers; atomic force microscopy; erythrocytes; community-acquired pneumonia.

Endogenous intoxication (El) syndrome is an obligatory sign of most acute infectious inflammatory processes including community-acquired pneumonia (CAP), and oftentimes determines the disease management and prognosis [1]. The main pathophysiological mechanisms of $\mathrm{El}$ is rapid accumulation of bacterial toxins, cellular debris, metabolites of oxidative stress, and other active substances in the body, as well as excessive proteolysis, against the background of developing inconsistency between excessive amount of toxic substances entering the tissues and the blood flow, and the capability of biological systems of body detoxification to transform, neutralize and eliminate these substances [2]. As clinical practice shows, most CAP patients have El manifestations dominating, which sometimes mask the symptoms of alveolar inflammation. Endotoxemia signs prevailing over the presentation of pulmonary tissue inflammation frequently occur in non-severe CAP, and in some cases it results in a late diagnosis. In severe CAP onset there are almost always the manifestations

For contacts: Vladimir N. Kotelnikov, e-mail 671235@mail.ru 
of respiratory failure and arterial hypoxemia, and EI symptoms just complement the clinical presentation. It is outside special endotoxemia variants: infectious-toxic shock and multi-organ failure, which most frequently develop against the background of septic conditions complicating pneumonia.

El severity is determined by a concentration level of bacterial and metabolic endotoxins in biological media of the body, which have a damaging effect on cell structures followed by a cascade of metabolic disorders. The following clinical laboratory tests are used in clinical practice to assess El severity: leukocyte (LII), hematological (HII), nuclear (NII) intoxication indices, and others [2]. Universal biochemical EI markers also include average-weight molecules (AWM), which are the result of excess proteolysis. Moreover, there are a few studies of cell nanostructure under endotoxemia. Modern biomedical technologies used for this purpose offer new opportunities to reveal pathophysiological regularities of the disease presentation in different groups of patients [3].

Atomic force microscopy (AFM) enables to record the force of interactions between two atoms (one located on the probe tip of the cantilever and the other on the sample surface) at a distance of over one angstrom, and use nanosizes that are effectively applied in studying the micro-relief and surface structure of biological tissues, fixes their 3D images at an atomic and molecular level that makes it possible to analyze the object nanostructure. In addition, using AFM we can measure elastic and adhesive properties of cell membranes closely connected with intra- and extracellular interactions [4]. AFM enables to form both geometrical "image" of a cell surface, as well as its physical and mechanical (biomechanical) "image" composed of a set of AFM parameters.

The aim of the investigation was to assess the information content of the parameters of atomic force microscopy to characterize the severity of endogenous intoxication in community-acquired pneumonia according to the findings of ultrastructural parameters of red blood cells.

Materials and Methods. We studied the examination findings of 71 male patients with CAP, who had undergone treatment in 439 Military Hospital, Ministry of Defence of the Russian Federation, in 2013-2014. Their age was $18-23$ years, mean age being $20.4 \pm 0.6$ years. All subjects were hospitalized on the first day of the disease, with no previous antibacterial therapy. In all cases CAP diagnosis was made according to microbiological, clinical and radiological and laboratory findings with due account for Russian Respiratory Society recommendations [5]. Non-severe CAP was diagnosed in 45 patients, and severe form - in 26 patients. A control group involved 20 healthy nonsmoking volunteers of the similar age.

The patients with non-severe CAP were characterized by the presence of focal inflammatory infiltrations in the lungs, which were subsegmental or within the bounds of several segments. The patients with severe CAP were found to have lobar, bilobar, or polysegmental bilateral pulmonary infiltrates, 8 patients among them were diagnosed to have parapneumonic exudative pleuritis. According to monitoring data of partial pressure of arterial oxygen $\left(\mathrm{PaO}_{2}\right)$ and blood oxygen saturation $\left(\mathrm{SpO}_{2}\right)$, this group of patients was recorded to have hypoxia, mean $\mathrm{PaO}_{2}$ being $90.71 \pm 0.68 \mathrm{Hg} \mathrm{mm}, \mathrm{SpO}_{2}-$ $89.62 \pm 0.87 \%$. The etiological structure of non-severe and severe CAP was the following: Streptococcus pneumoniae: 64.4 and $61.5 \%$, Haemophilus influenza: 17.8 and 23.1\%, Mycoplasma pneumonia: 11.1 and $11.5 \%$, Chlamydophila pneumonia: 4.4 and $7.7 \%$, respectively.

Due to clinical and laboratory findings characterizing endotoxemia severity [2], we distinguished three groups of the patients examined: group 1 involved 25 patients with El degree I, group 2 included 24 patients with EI degree II, and group 3 consisted of 22 patients with EI degree III. In addition, El degree I was typical only for patients with non-severe CAP, II-III degree was typical both for patients with severe CAP (26 patients), and also those with non-severe disease (20 patients).

Venous blood $(3 \mathrm{ml})$ was taken in all subjects within $24 \mathrm{~h}$ when admitted to hospital, and then on days 3, 7 and 14. Heparin $10 \mathrm{U} / \mathrm{ml}$ was used as an anticoagulant. Red blood cells were isolated by centrifugation within $10 \mathrm{~min}$ at 1,500 rpm. All patients underwent standard laboratory, microbiological and immunological investigations, hematological indices and intoxication indices (LII, HII, NII) being calculated. AWM concentration in blood plasma was determined [2]. A cytokine level in blood serum was measured by an enzyme immunoassay using test-systems to determine interleukin 10 (IL-10), tumor necrosis factor (TNF- $\alpha$ ) according to the technique of DuoSet ELISA Development Systems (R\&D Systems, Inc., USA).

Arterial blood gas was measured on an automatic analyzer ABL-725 (Radiometer Copenhagen, Denmark), $\mathrm{SpO}_{2}$ was determined using a pulse oximeter Nissei OX700 (Nihon Seimitsu Sokki Co., Ltd., Japan).

Red blood cell nanostructure was studied on a scanning atomic force microscope Bioscope Catalyst (Bruker, USA) in combination with an inverted microscope Axiovert 200 (Carl Zeiss, Germany). The images were taken in a contact Scan Asyst mode. To control sample damage in scanning, we selectively used a semi-non-contact mode. The sensors (cantilevers) used were: MSNL-10 (k: 0.07-0.107 N/m, $\left.\mathrm{f}_{0}: 15-33 \mathrm{kHz}\right)$ and SCANASYST-AIR ( $\mathrm{k}: 0.3-0.4 \mathrm{~N} / \mathrm{m}, \mathrm{f}_{0}: 54-92 \mathrm{kHz}$ ) (Bruker, USA).

To determine micromechanical characteristics of red blood cell membranes (elasticity modulus, elastic deformation (strain), adhesion force) we used a mode of nanomechanical mapping (PFQNM; Bruker, USA). Before every micromechanical mapping of a sample under study we calibrated a cantilever by 
reference cantilevers (Bruker, USA) with known surface mechanical properties. We determined the sensitivity to deviation in leucosapphire, elasticity modulus - by polyethylene: PDMS-SOFT1 (2.5 MPa) and PDMSSOFT2 (3.5 MPa). Moreover, we measured the cantilever point rounding radius on a special test wafer with titanium oxide coating. The calibrating findings using the program NanoScope v. 8.10 (Bruker, USA) were applied to calculate true elasticity constant $(k)$ and to image an object in micromechanical dimensions.

The study was carried out in accordance with the declaration of Helsinki (adopted in June 1964, Helsinki, Finland and revised in October 2000, Edinburgh, Scotland) and was approved by the Ethics Committee of Pacific State Medical University. All patients gave their written informed consent to process their personal data.

The experimental findings were processed by an analysis of variance using the analysis package Microsoft Excel 14. When comparing two independent groups we applied nonparametric Mann-Whitney test; the correlation was studied by a nonparametric technique of Spearman rank correlation analysis for nonnormal distribution.

Results and Discussion. For more precise ranging the patients by endotoxemia intensity and the following analysis of the effect of its factors on ultrastructural properties of red blood cells, we determined and calculated standard laboratory indices and intoxication indices (Table 1).

The dynamics of hematological indices demonstrated linear tendency associated with a reliable increase of their values with El augmentation. So, in group 1 LII, HII and NII values exceeded those of the control group by $1.6,4.2$ and 2.5 , respectively; in group 2: by $3.8,7.3$ and 6.3 times; in group 3 : by $12.9,28.8$ and 10 times. All patients of group 3 were recorded to have toxic granulation in white blood cells that also suggested severe endotoxemia. Moreover, there was found a significant AWM increase in blood plasma by $19.4 \%$ in El degree I, by $25.8 \%$ - in degree II, and by $58.1 \%$ - in degree III. In addition, severe endotoxemia was characterized by a significant (nearly by 1.6 times) increase of creatinine and urea concentration levels in blood serum.

Some works have defined a prognostic value of a cytokine level in developing CAP complications and outcomes [6]. There have been given evidences that the imbalance in cytokine system in acute inflammation provokes a cascade of metabolic disturbances related to the hyperproduction of active metabolites of oxygen, peroxynitrite, proteolysis products, that considerably determines the severity of a pathological process. The findings of our previous studies [7] showed IL-10 and TNF- $\alpha$ to be the most informative parameters of a cytokine status for El characteristic. The results of the present study demonstrate the significant (nearly by 20 times in relation to the control) cytokine concentration increase in severe El that enables to consider cytokines as immunological markers of the disease.

Thus, a complex assessment of El severity considerably completes a clinical characteristic of CAP patients, and specifies the idea of the intensity of metabolic disorders and functional state of natural biological detoxification systems.

At the next stage we studied erythrocyte membrane nanostructure in CAP patients with 3D imaging of some cells (Figure 1).

Color scale enables to estimate a cell profile and represent it in a graph form. The red blood cell diameter on the image is $6,816 \mathrm{~nm}$, the rim height is $530 \mathrm{~nm}$, the structural deflection discocyte is $138 \mathrm{~nm}$, the edge lift ordinate is $51.3 \mathrm{~nm}$. By setting the cursor in any direction, one can determine, with accuracy to $0.1 \mathrm{~nm}$, the required cell dimensions.

Table 1

Laboratory findings of endogenous intoxication in patients with community-acquired pneumonia $(\mathrm{M} \pm \mathrm{m})$

\begin{tabular}{|c|c|c|c|c|}
\hline \multirow[b]{2}{*}{ Parameters } & \multirow[b]{2}{*}{$\begin{array}{l}\text { Control } \\
(n=20)\end{array}$} & \multicolumn{3}{|c|}{ Endogenous intoxication severity } \\
\hline & & $\begin{array}{c}\text { Degree I } \\
\text { (group 1, } \\
n=25 \text { ) }\end{array}$ & $\begin{array}{c}\text { Degree II } \\
\text { (group 2, } \\
n=24 \text { ) }\end{array}$ & $\begin{array}{c}\text { Degree III } \\
\text { (group 3, } \\
n=22 \text { ) }\end{array}$ \\
\hline LII (CU) & $1.12 \pm 0.12$ & $1.81 \pm 0.21^{*}$ & $4.21 \pm 0.33^{v}$ & $14.28 \pm 1.69^{+}$ \\
\hline HII (CU) & $0.91 \pm 0.05$ & $3.81 \pm 0.29^{*}$ & $6.61 \pm 0.42^{v}$ & $26.02 \pm 2.61^{+}$ \\
\hline $\mathrm{NII}(\mathrm{CU})$ & $0.08 \pm 0.01$ & $0.22 \pm 0.05^{*}$ & $0.51 \pm 0.05^{v}$ & $0.81 \pm 0.09^{+}$ \\
\hline AWM (CU) & $0.31 \pm 0.02$ & $0.37 \pm 0.02^{*}$ & $0.39 \pm 0.03^{v}$ & $0.49 \pm 0.08^{v}$ \\
\hline $\begin{array}{l}\text { Creatinine } \\
(\mathrm{mmol} / \mathrm{L})\end{array}$ & $86.42 \pm 7.41$ & $93.01 \pm 3.12$ & $113.02 \pm 2.31^{*}$ & $138.52 \pm 9.71^{v}$ \\
\hline Urea (mmol/L) & $5.71 \pm 0.42$ & $6.21 \pm 0.23$ & $7.52 \pm 0.34$ & $9.41 \pm 0.39^{*}$ \\
\hline IL-10 (pkg/ml) & $4.25 \pm 2.41$ & $12.24 \pm 5.12^{*}$ & $19.38 \pm 6.13^{v}$ & $103.95 \pm 10.72^{v}$ \\
\hline TNF- $\alpha(p k g / m l)$ & $1.05 \pm 0.60$ & $0.96 \pm 0.62$ & $0.96 \pm 0.62$ & $23.65 \pm 3.62^{v}$ \\
\hline
\end{tabular}

Note. Significant difference of values with controls: ${ }^{*} p<0.05$; ${ }^{\vee} p<0.01$; ${ }^{+} p<0.001$. LII: leukocyte intoxication indices; HII: hematological intoxication indices; NII: nuclear intoxication indices; AWM: average-weight molecules.

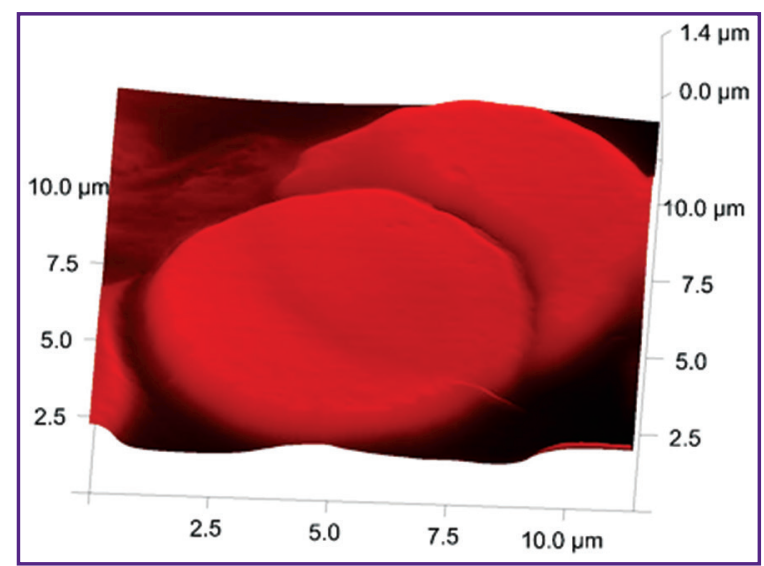

Figure 1. 3D image of single erythrocytes in communityacquired pneumonia 


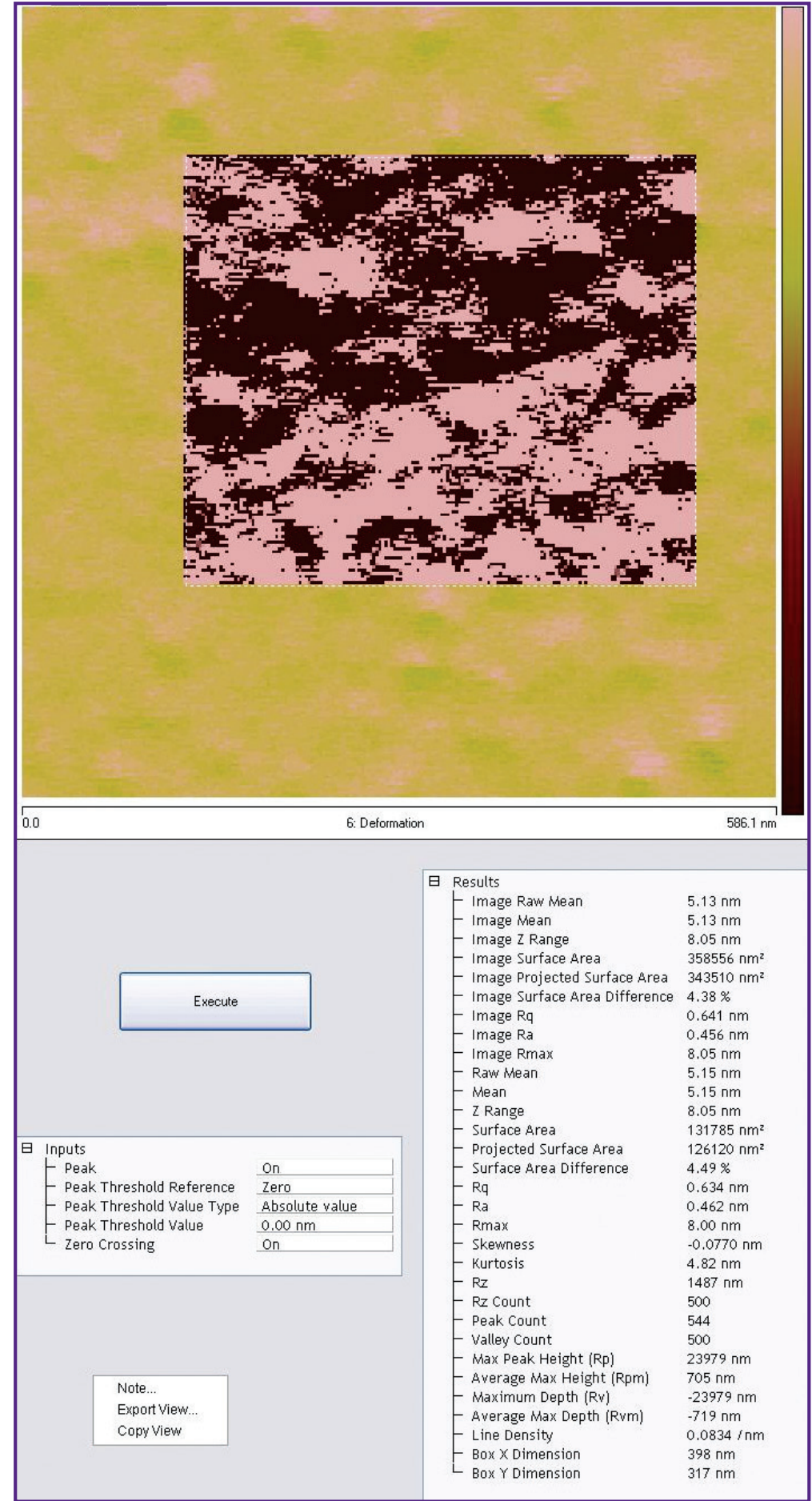

Figure 2. An example of the processing of findings of red blood cell membrane in terms of elastic deformation in degree III endogenous intoxication in patients with community-acquired pneumonia

Together with nanoanatomical parameters of erythrocytes, we studied micromechanical properties of the cells' membrane surface: elasticity modulus, adhesion force and elastic deformation (Figure 2).

Table 2 shows the obtained mean values of AFM parameters of red blood cells in El of different severity in CAP patients. Diameter, area, volume and other parameters of red blood cells describing their geometrical image were established to stay stable in all patients examined even in El degree III. The only altered parameters were those characterizing elastic properties of these cells. The intensity of the changes prevailed among CAP patients with EI degree III. So, the elasticity modulus of red blood cell membranes and adhesive force decreased by 5.2 and 2.4 times, respectively, while elastic deformation increased by 2.6 times. In patients with El degrees I and II these indices also significantly differed from the control values, though were less marked.

The correlation analysis findings demonstrate significant correlations between the parameters characterizing El severity and micromechanical properties of membranes (Table 3). In particular, there were found positive correlations of high intensity between LII ( $r=0.82)$, HII $(r=0.84)$, AWM $(r=0.88)$ and elastic deformation of red blood cells (in all cases: $p<0.01$ ). Strong feedback was revealed between LII ( $r=-0.84)$, HII $(r=-0.88)$, AWM $(r=-0.84)$ and elasticity modulus of red blood cell membrane, as well as its adhesion force. In addition, IL-10 and TNF- $\alpha$ showed strong relation with elastic deformation, and negative dependence on elasticity modulus and adhesion force of red blood cell membranes.

However, the analysis of these indices in patients with various CAP etiological structures did not reveal any quantitative differences and the directivity of changes of correlation relationship depending on an agent type.

In convalescents from groups 1 and 2 no divergences of red blood cell micromechanical parameters from control values were found. It suggests transient nature of the changes. Most group 3 patients appeared to maintain their significant differences from the norm, which were leveled as the follow-up showed 2.5-3 months after discharge from the hospital.

The study results showed that AFM parameters characterizing elastic properties of red blood cell membranes turned out to be the most sensitive to $\mathrm{El}$ in CAP. These parameters can considerably expand on clinical and laboratory characteristic of endotoxemia in pulmonary inflammatory disease.

Statistical processing of the findings enabled to calculate the reference values of micromechanical indices of red blood cell membranes in healthy young subjects and patients with $\mathrm{El}$ of different severity (Table 4). 
Table 2

Ultrastructural characteristics of red blood cells in endogenous intoxication in patients with community-acquired pneumonia $(\mathrm{M} \pm \mathrm{m})$

\begin{tabular}{|lcccc}
\hline \multicolumn{1}{|c}{ Red blood cell characteristics } & $\begin{array}{c}\text { Control } \\
(\mathrm{n}=20)\end{array}$ & $\begin{array}{c}\text { Degree I } \\
\text { (group 1, } \mathrm{n}=25)\end{array}$ & $\begin{array}{c}\text { Endogenous intoxication severity } \\
\text { (group 2, } \mathrm{n}=24)\end{array}$ & $\begin{array}{c}\text { Degree III } \\
\text { (group 3, } \mathrm{n}=22)\end{array}$ \\
\hline Diameter $(\mathrm{nm})$ & $6816.2 \pm 47.4$ & $\frac{6792.8 \pm 44.5}{6802.8 \pm 49.3}$ & $\frac{6788.1 \pm 41.9}{6812.1 \pm 47.8}$ & $\frac{6783.4 \pm 42.1}{6819.3 \pm 39.4}$ \\
\hline Rim height $(\mathrm{nm})$ & $530.7 \pm 15.4$ & $\frac{528.8 \pm 11.9}{531.2 \pm 12.7}$ & $\frac{528.8 \pm 11.9}{531.2 \pm 12.7}$ & $\frac{504.2 \pm 12.6}{522.7 \pm 16.2}$ \\
\hline Area $\left(\mu m^{2}\right)$ & $126.4 \pm 10.2$ & $\frac{121.3 \pm 10.4}{126.3 \pm 11.5}$ & $\frac{121.3 \pm 10.4}{126.3 \pm 11.5}$ & $\frac{117.6 \pm 11.9}{120.7 \pm 10.1}$ \\
\hline Volume $\left(\mu m^{3}\right)$ & $88.9 \pm 10.2$ & $\frac{91.7 \pm 9.9}{89.1 \pm 9.7}$ & $\frac{91.7 \pm 9.9}{89.1 \pm 9.7}$ & $\frac{101.0 \pm 10.5}{91.4 \pm 9.7}$ \\
\hline Structural deflection discocyte $(\mathrm{nm})$ & $138.3 \pm 9.4$ & $\frac{130.8 \pm 9.1}{138.1 \pm 10.4}$ & $\frac{130.8 \pm 9.1}{138.1 \pm 10.4}$ & $\frac{121.2 \pm 8.8}{134.7 \pm 9.4}$ \\
\hline Edge lift ordinate $(\mathrm{nm})$ & $88.9 \pm 10.1$ & $\frac{82.4 \pm 6.3}{89.1 \pm 7.8}$ & $\frac{82.4 \pm 6.3}{89.1 \pm 7.8}$ & $\frac{71.2 \pm 9.7}{84.4 \pm 8.2}$ \\
\hline Elasticity modulus $(\mathrm{MPa})$ & $34.0 \pm 2.1$ & $\frac{28.4 \pm 1.3^{*}}{34.1 \pm 1.1}$ & $\frac{21.5 \pm 1.4^{*}}{33.7 \pm 1.1}$ & $\frac{7.1 \pm 0.2^{* *}}{21.2 \pm 1.3^{*}}$ \\
\hline Elastic deformation $(\mathrm{nm})$ & $5.1 \pm 0.3$ & $\frac{6.4 \pm 0.1^{*}}{5.3 \pm 0.2}$ & $\frac{7.6 \pm 0.1^{*}}{5.8 \pm 0.2}$ & $\frac{13.2 \pm 1.1^{* *}}{7.4 \pm 0.5^{*}}$ \\
\hline Adhesion force $(\mathrm{nN})$ & $5.5 \pm 0.3$ & $\frac{4.3 \pm 0.1^{*}}{5.4 \pm 0.2}$ & $\frac{3.4 \pm 0.1^{*}}{5.2 \pm 0.2}$ & $\frac{2.3 \pm 0.1^{* *}}{4.1 \pm 0.3^{*}}$ \\
\hline
\end{tabular}

$\mathrm{N}$ o t e. Numerators represent the values before treatment, while denominators show the values after treatment. Significance of the differences before and after therapy: ${ }^{*} p<0.05$; ${ }^{* *} p<0.01$.

Table 3

Correlation coefficients of clinical and laboratory indices of endogenous intoxication and AFM parameters in patients with community-acquired pneumonia

\begin{tabular}{lcccccccc}
\hline \multirow{2}{*}{ AFM parameters } & \multicolumn{7}{c}{ Laboratory biochemical indices } \\
& IL-10 & TNF- $\alpha$ & LII & NII & HII & AWM & Creatinine & Urea \\
\hline Elasticity modulus & $-0.84^{*}$ & $-0.74^{*}$ & $-0.84^{* *}$ & $-0.73^{*}$ & $-0.88^{* *}$ & $-0.84^{*}$ & -0.49 & $-0.64^{*}$ \\
\hline Elastic deformation & $0.85^{*}$ & $0.73^{*}$ & $0.82^{*}$ & 0.54 & $0.82^{*}$ & $0.88^{*}$ & 0.43 & 0.35 \\
\hline Adhesion force & $-0.88^{*}$ & $-0.74^{*}$ & $-0.83^{*}$ & $-0.74^{*}$ & $-0.81^{*}$ & $-0.83^{*}$ & $-0.65^{*}$ & 0.46 \\
\hline
\end{tabular}

$\mathrm{N}$ o t e. * $\mathrm{p}<0.05 ;{ }^{* *} \mathrm{p}<0.01$. LII: leukocyte intoxication indices; NII: nuclear intoxication indices; HII: hematological intoxication indices; AWM: average-weight molecules.

The represented values can serve as a guide mark in interpreting AFM parameters and similar samplings in further studies including those to monitor the efficiency of endotoxemia pharmacocorrection and recovery completeness.

AFM technique is used to assess a condition of various objects, however, there are just a few works, which have applied the method to study cell surface in CAP [8, 9]. In addition, red blood cell membrane for a long time has been considered by researchers as a permeable membrane only for blood gases and as a membrane to separate hemoglobin from plasma. However, the progress achieved in biomaterial science makes it possible to consider red blood cell membrane to be the most
Table 4

Reference values of AFM parameters of red blood cells in healthy young subjects and patients with endogenous intoxication in community-acquired pneumonia

\begin{tabular}{lcccc}
\hline \multicolumn{1}{c}{ Parameters } & \multirow{2}{*}{ Norm } & \multicolumn{3}{c}{ Endogenous intoxication severity } \\
\cline { 3 - 5 } & & Degree I & Degree II & Degree III \\
\hline Elasticity modulus $(\mathrm{MPa})$ & $30.6-37.4$ & $30.5-23.8$ & $23.7-7.2$ & $<7.2$ \\
\hline Elastic deformation $(\mathrm{nm})$ & $4.6-5.6$ & $5.7-6.6$ & $6.7-13.2$ & $>13.2$ \\
\hline Adhesion force $(\mathrm{nN})$ & $5.0-6.1$ & $6.2-3.9$ & $3.8-2.3$ & $<2.3$ \\
\hline
\end{tabular}

important cell element, which is both a mechanical membrane with controlled physical properties, and also a central element exercising the coordination of 
cell functioning due to physical and chemical signals incoming to a cell [4].

Detoxification activity of red blood cells associated with utilization of endogenous aldehydes forming during lipid peroxidation, in glycosylation and in the oxidation of radicals of some free amino acids takes a key role under endotoxemia in CAP [2]. At the same time, carbohydrates localized in glycocalyx are responsible for adhesive properties of red blood cells, and are specific receptors to bind various ligands, viruses, antibodies. The decrease of elasticity modulus and adhesion force values of erythrocyte membrane revealed in the present study can indicate an adaptive nature of the reaction resulting in increased cell plasticity under impaired microcirculation and hypoxemia. Circulating red blood cells due to their elasticity assume various configurations adapting to the vessel shape. Thereby, capability to deformation is to be considered as one of the basic properties of erythrocytes. In the norm, red blood cells exhibit high deformability due to membrane elasticity and low viscosity of intracellular content. The increase of 'flowability' of a lipid biolayer can contribute to the growth of spare capacities to rapid recovery of membrane integrity in case of slight damage under impaired microcirculation during severe El [10]. It is certain to enhance the efficiency of gas transportation function of red blood cells. However, in a number of cases in severe endotoxemia in CAP patients, as well as in elderly patients with comorbidities, continued decrease in red blood cell membrane elasticity can serve as a certain trigger initiating a cascade of irreversible ultrastructural alterations of blood cells resulting in damaging their cytoskeleton and gas-transport dysfunction [3]. Thus, in young people transient changes of micromechanical alterations of red blood cell membrane indicate the unique plasticity of these cells and their high adaptive potential that enables to utilize these parameters as complementary markers of endotoxemia severity.

Conclusion. The studies prove the information content of micromechanical parameters of erythrocyte membranes by atomic force microscopy to characterize the severity of endogenous intoxication in communityacquired pneumonia. The findings enable to complement the notion of pathophysiological sequelae of endogenous intoxication, their effect on cell ultrastructure and adaptive nature of changes of micromechanical properties of their membranes.
Study Funding. The study was not funded by any sources.

Conflicts of Interest. The authors have no conflicts of interest related to the present study.

\section{References}

1. Musher D.M., Thorner A.R. Community-acquired pneumonia. N Engl J Med 2014; 371(17): 1619-1628, https:// doi.org/10.1056/nejmra1312885.

2. Borodin E.A., Egorshina E.V., Samsonov V.P. Biokhimiya endotoksikoza. Mekhanizmy razvitiya $i$ otsenka stepeni tyazhesti pri vospalitel'nykh zabolevaniyakh legkikh [Endotoxemia biochemistry. The mechanisms of development and severity assessment in pneumonia]. Blagoveshchensk: AGMA; 2003; 129 p.

3. Moeendarbary E., Harris A.R. Cell mechanics: principles, practices, and prospects. Wiley Interdiscip Rev Syst Biol Med 2014; 6(5): 371-388, https://doi.org/10.1002/wsbm.1275.

4. Zabinyakov N.A., Dovgiy P.G., Poltoratskiy A.N., Azarov K.S. Structural and functional parameters of erythrocytes and neutrophils from patients of middle, elderly and senile age with acute pneumonia. Gerontologiya 2014; 2(1): 23-33.

5. Chuchalin A.G., Sinopal'nikov A.l., Kozlov R.S., Avdeev S.N., Tyurin I.E., Rudnov V.A., Rachina S.A., Fesenko O.V. Clinical guidelines on diagnosis, treatment and prevention of severe community-acquired pneumonia in adults. Russian Pulmonology 2014; 4: 13-48.

6. Kondrashova N.M., Plekhova N.G., Somova L.M., Kostyushko A.V., Geltser B.I. Optimising estimation of parameters describing functional activity of innate immune cells in case of inflammatory lung diseases. Tikhookeanskiy meditsinskiy zhurnal 2012; 1: 83-86.

7. Geltser B.I., Kim A.P., Kotelnikov V.N., Makarov A.B. Features of immune response in patients with communityacquired pneumonia with endogenous intoxication of different degrees of severity. Tsitokiny i vospalenie 2015; 14(3): 35-41.

8. Proshchaev K.I., Zabinyakov N.A., Azarov K.S., Dovgii P.G. Studies of the structure and functions of blood cells in senile patients with pneumonia on the biological model of hypoxia by scanning probe microscopy. Bull Exper Biol 2014; 158(2): 256-259, https://doi.org/10.1007/s10517-014-2735-2.

9. Lamzin I.M., Khayrullin R.M. The quality assessment of stored red blood cells probed using atomic-force microscopy. Anat Res Int 2014; 2014: 869683, https://doi. org/10.1155/2014/869683.

10. Ivanov K.P. Modern medical problems of microcirculation and hypoxic syndrome. Vestnik Rossiiskoi akademii meditsinskikh nauk 2014; 69(1-2): 57-63, https://doi. org/10.15690/vramn.v69.i1-2.943. 